\title{
Betulinic acid increases radiosensitization of oral squamous cell carcinoma through inducing Sp1 sumoylation and PTEN expression
}

\author{
DAO-YING YUAN ${ }^{1,2}$, ZHEN MENG $^{2}$, KAI XU $^{2}$, QIAO-FENG LI ${ }^{2}$, \\ CHENG CHEN $^{2}$, KE-YI LI ${ }^{2}$ and BIN ZHANG ${ }^{1,2}$ \\ ${ }^{1}$ School of Stomatology, Shandong University, Jinan; ${ }^{2}$ Department of Oral and Maxillofacial Surgery, \\ Liaocheng People's Hospital, Liaocheng, Shandong 252000, P.R. China
}

Received August 22, 2016; Accepted June 2, 2017

DOI: $10.3892 / o r .2017 .5872$

\begin{abstract}
Radiotherapy is one of the most effective nonsurgical treatments for oral squamous cell carcinoma. However, radioresistance remains a major impediment to radiotherapy. Although BetA (Betulinic acid) can induce radiosensitization, the underlying mechanism and whether it could induce radiosensitization in oral squamous cell carcinoma are not fully understood. In this study, we showed that BetA increased radiosensitization in CAL-27 and Tca-83 cells. Radiation-triggered Sp1 overexpression was responsible for radioresistance of OSCC (oral squamous cell carcinoma) cells. Treatment with BetA downregulated Sp1 and upregulated PTEN through inducing Sp1 sumoylation and correspondingly increased radiosensitization. Moreover, Sumoylation of Sp1 upregulated PTEN protein expression by downregulating Sp1 as well as inhibiting Sp1 DNA binding activity, thereby leading to the activation of PTEN transcription. Our results suggested that BetA was able to enhance radiosensitization at least partially by downregulating Sp1 and upregulating PTEN through inducing Sp1 sumoylation. BetA is suggested to be a promising drug for increasing radiosensitization in oral squamous cell carcinoma radiotherapy.
\end{abstract}

\section{Introduction}

Oral squamous cell carcinoma (OSCC) accounts for approximately $3 \%$ of all newly diagnosed cancer cases, and is the most common head and neck cancer (1). Despite modern

Correspondence to: Professor Bin Zhang or Dr Ke-Yi Li, Department of Oral and Maxillofacial Surgery, Liaocheng People's Hospital, 67 Dongchangxi Road, Liaocheng, Shandong 252000, P.R. China

E-mail: zhangbin252000@163.com

E-mail:1kyxyw@163.com

Key words: radiation, betulinic acid, sumoylation, Sp1, PTEN, oral squamous cell carcinoma improvements in the treatment modalities, the 5-year overall survival rate of OSCC patients is still approximately $50 \%$ (2). In the United States, there are approximately 32,000 newly diagnosed OSCC cases and OSCC is likely responsible for an estimated 6,500 deaths in 2016 (3), while in China, the newly diagnosed and death cases was 48,000 and 22,100, respectively in 2015 (4). Therefore, it is critical to improve clinical outcomes of OSCC.

New therapeutic strategies targeting molecules critical for OSCC have shown promise in recent years (5-7), however, radiotherapy is still one of the most effective non-surgical treatments for tumors $(8,9)$. As an important treatment for cancers, radiation inhibits tumor growth, promotes tumor cell apoptosis, and prolongs patient survival (10). However, radioresistance remains a major impediment to radiotherapy $(11,12)$. Cancer cells resistant to radiotherapy can result in local recurrence (13). Exposure of cancer cells to radiation can activate several signal pathways that lead to radiation resistance, including Nuclear factor- $\kappa \mathrm{B}(\mathrm{NF}-\kappa \mathrm{B})$ and the signal transducer and activator of transcription 3 (STAT3) (14-16). DNA-dependent protein kinase catalytic subunit (DNA-PKcs) and ataxia telangiectasia-mutated gene (ATM) induce radioresistance by triggering reparation of radiation-induced DNA damage $(17,18)$. Specificity factor $1(\mathrm{Sp} 1)$ was also reported to be involved in radioresistance (19-21), therefore, Sp1 may serve as a target for increasing cancer radiosensitivity.

Sp1 plays multiple roles in several cellular processes, including cell growth, differentiation and apoptosis (22). Sp1 is overexpressed in many types of tumors, such as in breast cancers, pancreatic tumors, thyroid tumors, gastric tumors, liver cancers and gliomas (23-25), and is a negative prognostic factor for survival. The transcriptional activity of $\mathrm{Sp1}$ is modulated by several post-translational modifications, such as acetylation (26), phosphorylation (27), sumoylation (28), and $O$-GlcNAcylation (29). Post-translational modification can regulate protein level, transactivation activity, or DNA binding affinity of Sp1 (30). Sp1 is a ubiquitous transcription factor and functions by binding to the promoter of its target genes, including MMP3, MMP9, and cyclin D1. We have previously demonstrated that $\mathrm{Sp} 1$ could downregulate PTEN expression by binding to the specific site on PTEN promoter (26), but 
whether Sp1/PTEN participate in radiosensitization remains unknown.

PTEN (Phosphatase and tension homolog deleted on chromosome ten) is an important tumor-suppressor gene (31) and a dual-specificity phosphatase that removes phosphates from both proteins and lipids (31). PTEN antagonizes the PI3K-AKT signaling pathway (32). Mutation, deletion or dysfunction of PTEN was found in many types of tumors, such as breast cancers, glioblastomas (33), prostate cancers, thyroid cancers and endometrial carcinomas (33). PTEN acts as a pivotal determinant in regulating radio-response of cancer cells. Several genes, miRNAs or drugs regulate radioresistance through PTEN. Activation of PTEN by COX-2 inhibitors could induce cancer cell radiosensitivity (34). miRNA29a, miRNA21, miRNA16b could regulate radiosensitivity through targeting PTEN (35-37). PTEN/PI3K/AKT pathways were demonstrated mediating radiosensitivity (35). PTEN/Akt/HIF-1 $\alpha$ feedback loop modulates miRNA210induced radiosensitivity (37). Ionizing radiation induces EMT (epithelial-mesenchymal transition) through inhibiting PTEN and correspondingly activating Akt/GSK-3 $\beta /$ Snail signaling (38). Thus, whether Sp1 could regulate radiosensitivity by targeting PTEN needs further study. Betulinic acid (3 $\beta$, hydroxy-lup-20 (29)-en-28-oic acid; BetA) is a naturally occurring pentacyclic triterpenoid found in many kinds of fruits, vegetables and most abundant in the Sambucus williamsii Hance tree $(39,40)$. Betulinic acid has anti-malarial, anti-HIV and anti-inflammatory activities (41). BetA has also been found to have anticancer activities in various cancers through inducing apoptosis and inhibition of cell proliferation in several cancers, including pancreatic cancer, prostate cancer, leukemia and melanoma (42-47). BetA could regulate the expression or post-translational modification of lamin $\mathrm{B} 1, \mathrm{Sp} 1, \mathrm{NF}-\kappa \mathrm{B}$ and result in cell proliferation inhibition and apoptosis (46-48). Furthermore, BetA exerts anticancer activity through selectively inhibiting the growth of cancer cells but without affecting the normal cells $(41,49)$. Therefore, Bet A is a promising anticancer candidate. However, whether BetA could be used increasing radiosensitization in OSCC was unknown.

In this study, we examined i) whether BetA could induce radiosensitization in $\mathrm{OSCC}$, ii) whether Sp1 involved in radioresistance in OSCC and iii) whether BetA induced radiosensitization through $\mathrm{Sp} 1$ and the underlying mechanism.

\section{Materials and methods}

Cell culture and treatments. CAL-27 cells were incubated in Dulbecco's modified Eagle's medium (Gibco, Grand Island, NY, USA) with $10 \%$ fetal bovine serum (FBS) at $37^{\circ} \mathrm{C}$ with $5 \% \mathrm{CO}_{2}$. Tca-83 cells were incubated in RPMI-1640 medium (Gibco) with $10 \% \mathrm{FBS}$ at $37^{\circ} \mathrm{C}$ with $5 \% \mathrm{CO}_{2}$. Cells were exposed to radiation (Cobalt-60) at different doses at the dose rate of $2.544 \mathrm{~Gy} / \mathrm{min}$ and then cultured for $24 \mathrm{~h}$. Cells were also exposed to BetA alone for $24 \mathrm{~h}$. In another treatment, cells were exposed to a combination of radiation and BetA; first, cells were treated with BetA and then with radiation.

Plasmids, reagents and antibodies. Wild-type or mutant PTEN promoter-reporters were constructed in our previous

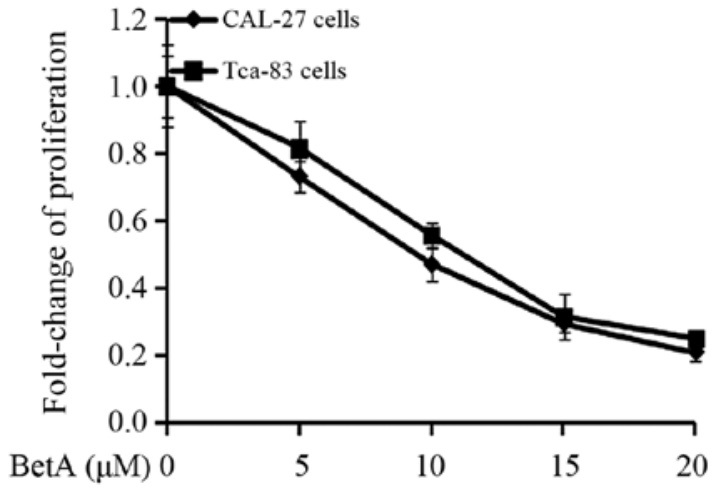

Figure 1. CAL-27 cells and Tca- 83 cells were exposed to different dose of BetA, and cell proliferation were measured with MTT assay.

study (26). The pcDNA3.0-SUMO-1 plasmid (Plasmid \#21154) was obtained from Addgene. BetA was purchased from Selleck Chemicals (Houston, TX, USA). Anti-PTEN (\#9552) antibody, anti-cleaved caspase 3 antibody (\#9661) were purchased from Cell Signaling Technology (Danvers, MA, USA). SUMO-1 siRNA (sc-29498), anti- $\beta$-actin (I-19) antibody (sc-1616) and anti-SUMO-1 antibody (sc-9060) were purchased from Santa Cruz Biotechnology (Santa Cruz, CA, USA). Anti-Sp1 antibody (07-645) was purchased from Merck Millipore (Billerica, MA, USA).

Protein extraction and western blotting. Whole cell lysates were extracted with RIPA lysis buffer (Applygen Technologies, Inc., Beijing, China). Protein concentrations were measured with BCA protein assay (Thermo Fisher Scientific, Waltham, MA, USA). Equal amounts of samples were subjected to $10 \%$ sodium dodecyl sulfate (SDS)-polyacrylamide gel electrophoresis (PAGE) and transferred onto a nitrocellulose membrane (Millipore). The membrane was blocked with 5\% fat-free milk in TBS-T (Tris buffer saline-Tween-20) for $1 \mathrm{~h}$. After incubation with primary antibodies diluted, 1:1,000 in TBS-T containing $1 \%$ milk overnight at $4^{\circ} \mathrm{C}$, the membrane was extensively washed with TBS-T thrice and then incubated with a secondary antibody conjugated with fluorophore for $1 \mathrm{~h}$ at room temperature. After extensively washed thrice with TBS-T, the membrane was visualized with Odyssey infrared imaging system (Odyssey LI-COR). For internal controls of equal loading, the blots were also stripped and reprobed with $\beta$-actin antibody.

Luciferase assay. Luciferase assay was performed as previously described (26). Briefly, PTEN reporter plasmid (1 $\mu \mathrm{g})$ was transfected with Lipofectamine 2000 (Invitrogen Life Technologies, Carlsbad, CA, USA) into CAL-27 cells in a 12 -well plate. The transfected cells were lysed in a cell lysis buffer $24 \mathrm{~h}$ after transfection. Luciferase activity was measured with an LB960 microplate luminometer (Berthold) using luciferin as the substrate, according to the manufacturer's instructions (Promega Corp., Madison, WI, USA).

Assessment of cell apoptosis. Cells were washed with phosphate-buffered saline (PBS) thrice, fixed with $10 \%$ formaldehyde for $5 \mathrm{~min}$, and incubated with $5 \mathrm{mg} / \mathrm{ml}$ 

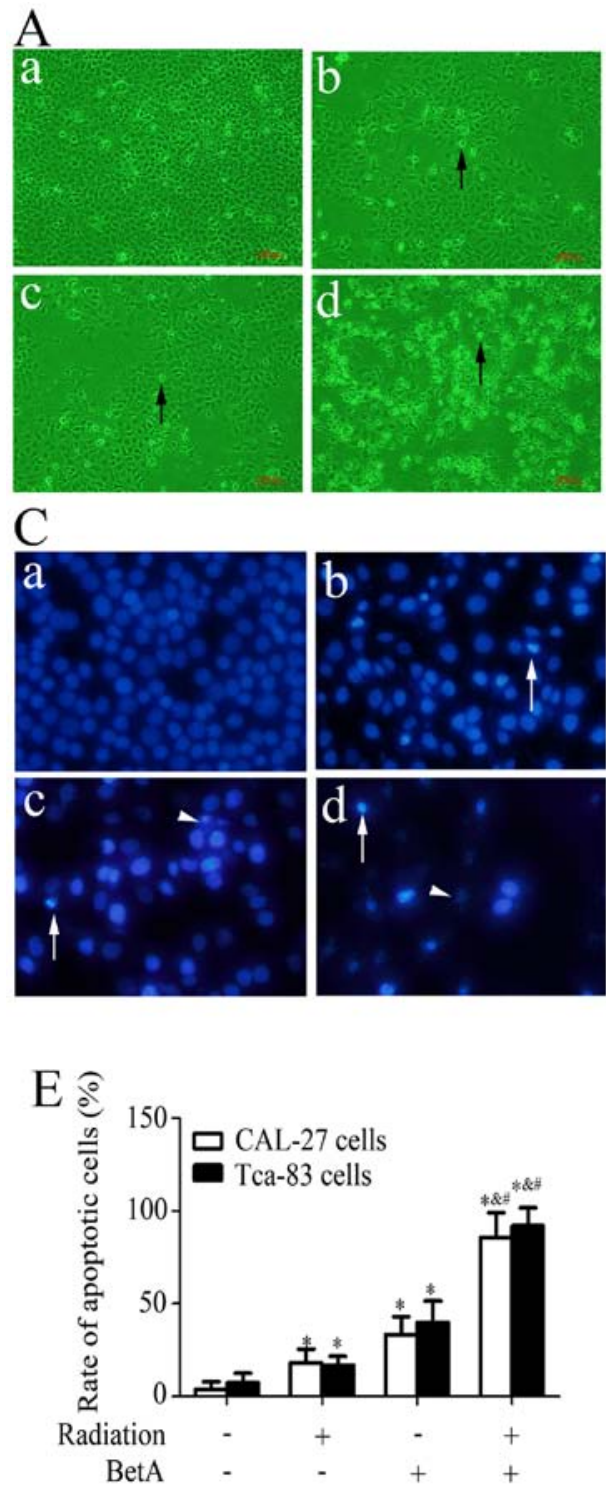

B
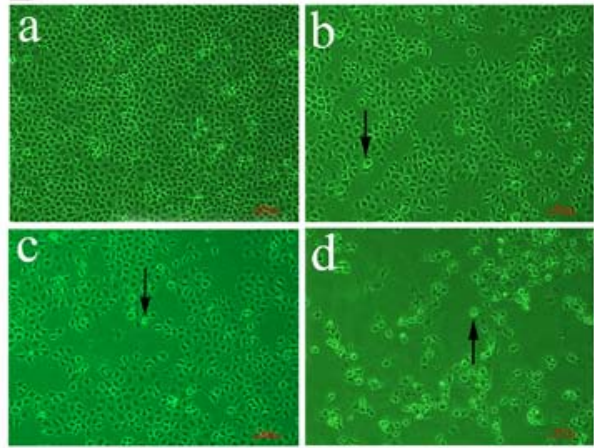

D
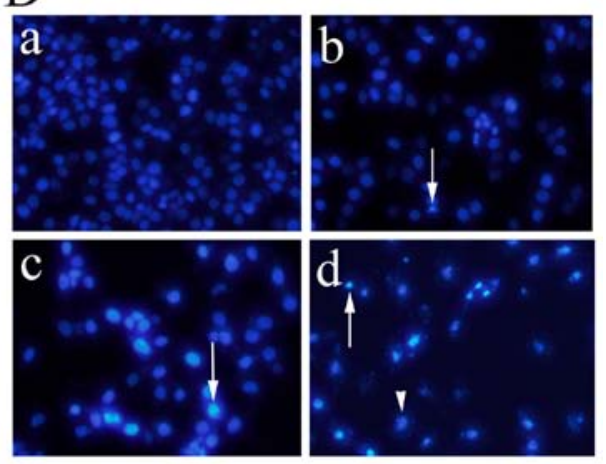

F

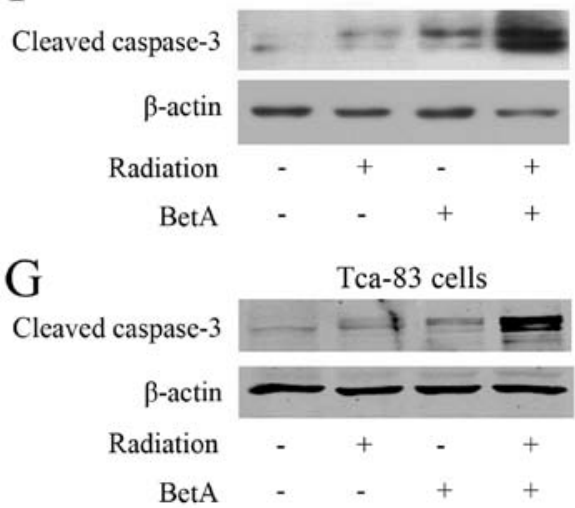

Figure 2. BetA enhances radiation-induced inhibition of cell proliferation and apoptosis. (A and B) Microphotographs of cells after different treatments. (A) CAL-27 cells and (B) Tca-83 cells were treated with radiation $(8 \mathrm{~Gy})$, BetA $(10 \mu \mathrm{M})$ or a combination of radiation and BetA. Apoptotic cells were shrunken and floating (arrows). (C and D) Fluorescent microphotographs of cells after different treatments. (C) CAL-27 cells and (D) Tca-83 cells were treated with radiation, BetA or a combination of radiation and BetA. Cells were stained with DAPI after different treatments and those with nuclear condensation (arrows) or fragmentation (triangle) were identified as apoptotic cells. (E) Quantity of cell apoptosis rate. Data are presented as mean \pm SD of at least three independent experiments. ${ }^{*} \mathrm{P}<0.05$ vs. the control group; ${ }^{\&} \mathrm{P}<0.05$ vs. the radiation group; ${ }^{\#} \mathrm{P}<0.05$ vs. the Bet $\mathrm{A}$ group ( $\mathrm{n}=3$ ). a, Control group; $\mathrm{b}$, radiation group; $\mathrm{c}, \mathrm{Bet} \mathrm{A}$ group; d, radiation and BetA group. (F) CAL-27 cells and (G) Tca-83 cells were treated with radiation, BetA or a combination of radiation and BetA, and the cleaved caspase-3 was detected using western blot analysis.

4',6-diamidino-2-phenylindole dihydrochloride (DAPI) in the dark for 3 min at room temperature. After washed with PBS, the cells were examined under a fluorescence microscope (Nikon Corporation, Tokyo, Japan). The cells presenting features of nuclear condensation and fragmentation were identified as apoptotic cells and were counted within the six randomly selected fields. The rate of apoptotic cells was presented as mean \pm SD of at least three independent experiments.

Immunoprecipitation. Whole-cell extracts $(2.5 \mathrm{mg})$ were incubated in $500 \mu \mathrm{l}$ extraction buffer with $4 \mu \mathrm{g}$ anti-Sp1 antibody for $16 \mathrm{~h}$ at $4^{\circ} \mathrm{C}$, added with $40 \mu \mathrm{l}$ protein $\mathrm{A} / \mathrm{G}$-agarose beads
(Santa Cruz Biotechnology), incubated again for $1 \mathrm{~h}$ at $4^{\circ} \mathrm{C}$ and then washed five times with extraction buffer. The bound proteins were released by boiling in a loading buffer and then subjected to immunoblot analysis. Sumoylated Sp1 was detected using anti-SUMO-1 antibody. The membranes were stripped for Sp1 detection.

Chromatin immunoprecipitation assay. Chromatin immunoprecipitation (ChIP) assay was performed using a ChIP assay kit (Upstate). Briefly, CAL-27 cells were cross-linked with $1 \%$ formaldehyde. The chromatin was sonicated into fragments ranging between 200 and 1000 bp and then was pulled down by 

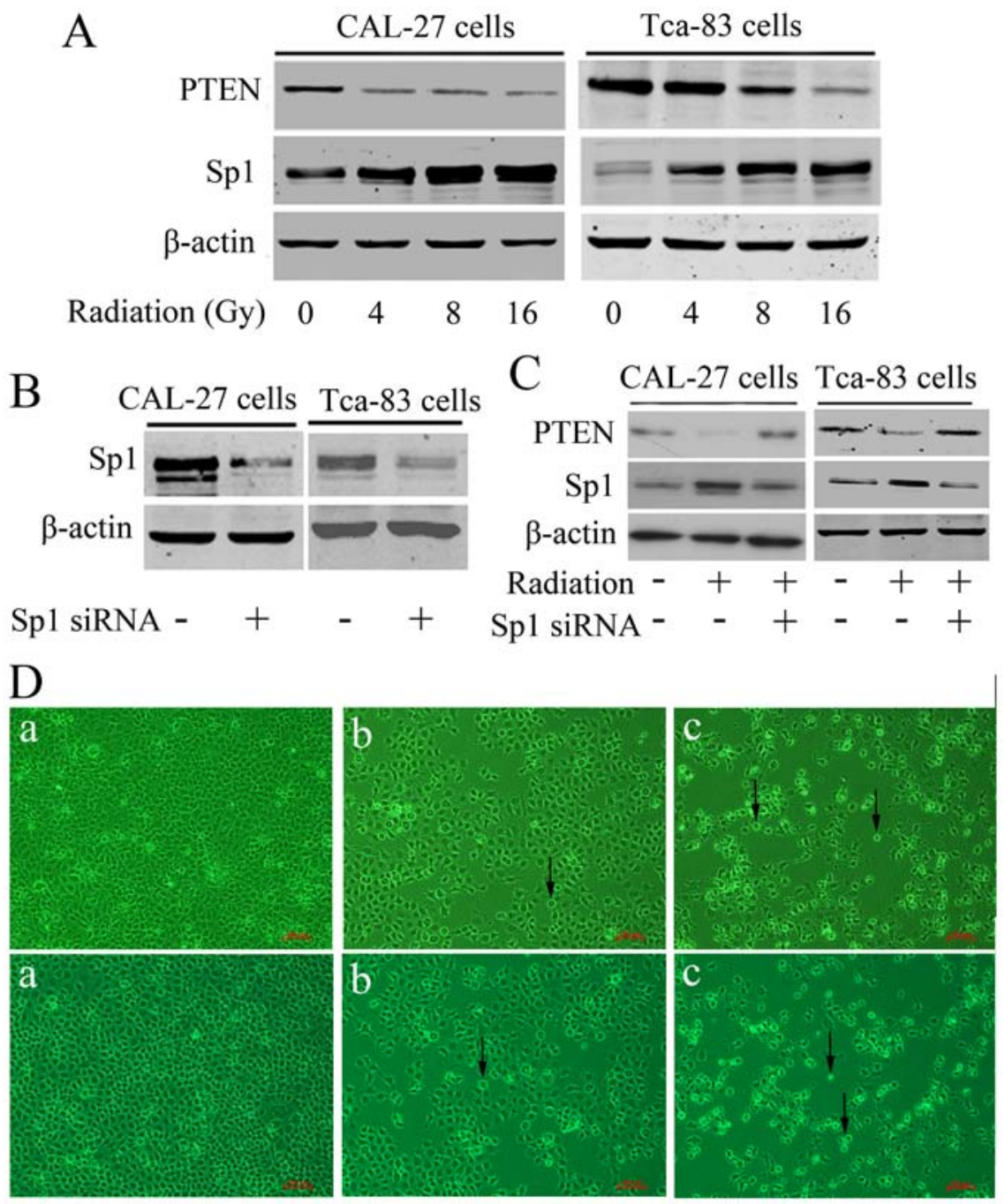

Figure 3. Sp1 may be responsible for radioresistance of OSCC. (A) Western blot analysis of Sp1 and PTEN after radiation. CAL-27 (left panel) and Tca-83 cells (right panel) were lysed and subjected to western blotting after exposure to increasing doses of radiation. (B) Confirmation of knockdown of Sp1. CAL-27 (left panel) and Tca-83 cells (right panel) were transfected with scrambled siRNA or Sp1 siRNA for $48 \mathrm{~h}$. Protein expression was detected by western blot analysis. (C) Knockdown of Sp1 antagonized radiation-induced PTEN downregulation. CAL-27 (left panel) and Tca-83 cells (right panel) were transfected with scrambled siRNA or Spl siRNA for $48 \mathrm{~h}$, and then exposed to radiation. Protein expression was detected by western blot analysis. (D) Microphotographs of cells after different treatments. CAL-27 (upper panel) and Tca-83 cells (lower panel) were transfected with scrambled siRNA or Sp1 siRNA for $48 \mathrm{~h}$, and then exposed to radiation. Apoptotic cells were shrunken and floating (arrows). a, Control group; b, radiation group; c, Sp1 siRNA and radiation group.

anti-Sp1 antibody for PCR amplification. The primers for amplifying the fragments (-1138 to -606) containing Sp1-binding site of the PTEN promoter are as follows: 5'-AGGCAGCTACA CTGGGCAT-3' (sense) and 5'-AGGAAGAGGCTGCACGG TTAGAAA-3' (antisense). The PCR products were analyzed on $1.5 \%$ agarose gel and then photographed

Statistical analysis. Statistical analysis was performed using SPSS 11.5 for Windows. The data are presented as mean \pm SD. Differences between multiple groups were analyzed by one-way analysis of variance. A value of $\mathrm{P}<0.05$ was considered to indicate a statistically significant difference.

\section{Results}

BetA enhances radiation-induced inhibition of cell proliferation and apoptosis. To examine whether BetA could enhance radiosensitization of OSCC, OSCC cells were treated with radiation or BetA alone or a combination of radiation and BetA. First of all, we determined the dose of BetA. We performed an MTT assay and found that $10 \mu \mathrm{M}$ of BetA could cause approximately 50\% proliferation inhibition of CAL-27 cells and Tca83 cells (Fig. 1); furthermore, we found that this concentration of BetA only slightly promoted apoptosis of CAL-27 cells and Tca- 83 cells, while combined with radiation, it significantly induced apoptosis of these two cells (Fig. 2C-E). Actually, this concentration of BetA was used also by some other teams in the study of BetA inducing radiosensitization $(50,51)$. Therefore, we chose the concentration of $10 \mu \mathrm{M}$.

Two OSCC cell lines, CAL-27 and Tca-83 cells, were exposed to radiation with $8 \mathrm{~Gy}$ at the dose rate of $2.544 \mathrm{~Gy} / \mathrm{min}$ or $10 \mu \mathrm{M}$ BetA alone or a combination of radiation and BetA for $24 \mathrm{~h}$. As shown in Fig. 2A-E, treatment with radiation or BetA alone resulted in only a slight inhibition of cell proliferation and a slight increase in apoptosis, whereas treatment with radiation after BetA treatment significantly enhanced 

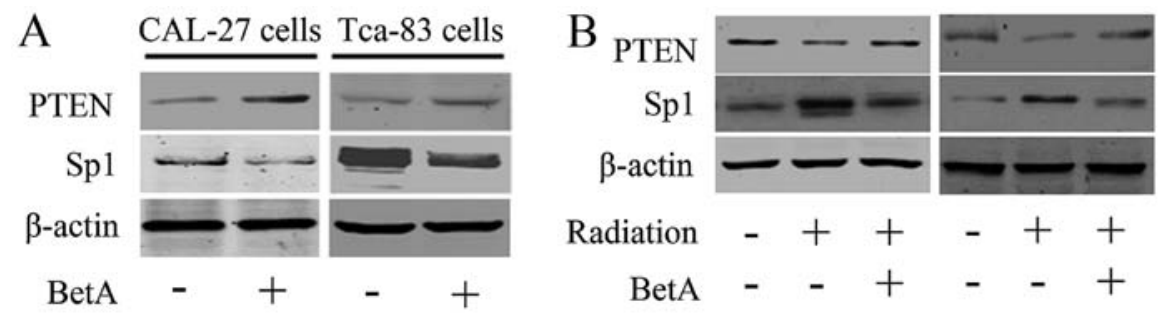

Figure 4. BetA antagonized radiation-induced Sp1 upregulation and rescued PTEN expression. (A) BetA inhibited Sp1 expression and induced PTEN expression. CAL-27 (left panel) and Tca-83 cells (right panel) were treated with $10 \mu \mathrm{M}$ BetA and then protein expression was detected by western blot analysis (B) BetA antagonized radiation-induced Sp1 upregulation and rescued PTEN expression. CAL-27 (left panel) and Tca-83 cells (right panel) were treated with $10 \mu \mathrm{M}$ BetA and then exposed to radiation. Protein expression was detected by western blot analysis.

A

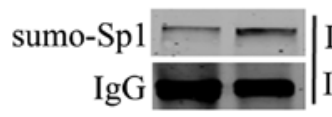
IP:anti-Spl IB:anti-SUMO-1

pcDNA3.0-SUMO-1 - $\quad+$

$\mathrm{C}$ \begin{tabular}{r|l} 
sumo-Sp1 & IP:anti-Sp1 \\
IgG $=$ & IB:anti-SUMO-1
\end{tabular}

SUMO-1 SIRNA - +

$\mathrm{E}$

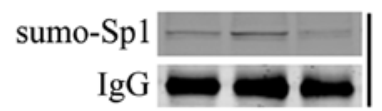

IP:anti-Sp1 IB:anti-SUMO-1

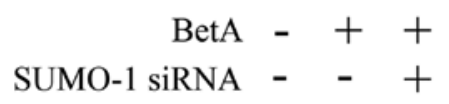

B

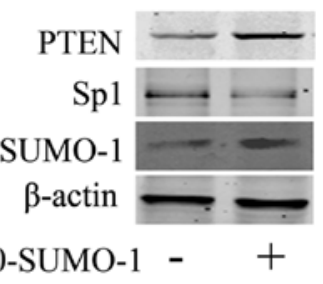

D

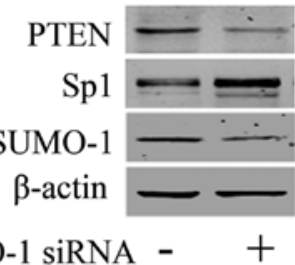

F

PTEN

$\mathrm{Sp} 1$

SUMO-1

$\beta$-actin

BetA - + +

SUMO-1 SiRNA - $\quad$ - +

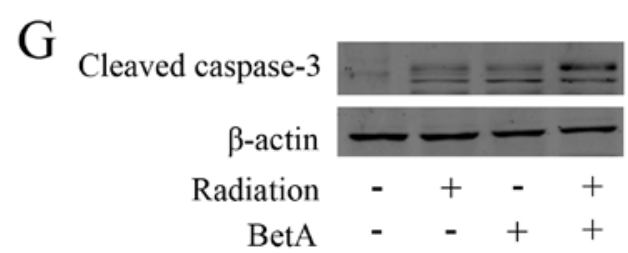

Figure 5. BetA inhibits Sp1 expression through inducing Sp1 sumoylation. (A) SUMO-1 transfection increased Sp1 sumoylation. pcDNA3.0-SUMO-1 was transfected into CAL-27 cells for $48 \mathrm{~h}$. Whole-cell lysates were immunoprecipitated with Sp1 antibody and subjected to western blotting with anti-SUMO-1 antibody. (B) SUMO-1 transfection decreased Sp1 expression and increased PTEN expression. pcDNA3.0-SUMO-1 was transfected into CAL-27 cells for 48 h. Protein expression was detected by western blot analysis. (C) Knockdown of SUMO-1 decreased Sp1 sumoylation. SUMO-1 siRNA was transfected into CAL-27 cells for $48 \mathrm{~h}$. Whole-cell lysates were immunoprecipitated with Sp1 antibody and subjected to western blotting with anti-SUMO-1 antibody. (D) Knockdown of SUMO-1 increased Spl expression and decreased PTEN expression. SUMO-1 siRNA was transfected into CAL-27 cells for 48 h. Protein expression was detected by western blot analysis. (E) SUMO-1 knockdown antagonized BetA-induced Sp1 sumoylation. SUMO-1 siRNA was transfected into CAL-27 cells for $24 \mathrm{~h}$ and then treated with BetA $(10 \mu \mathrm{M})$ for another $24 \mathrm{~h}$. Whole-cell lysates were immunoprecipitated with Spl antibody and subjected to western blotting with anti-SUMO-1 antibody. IP, immunoprecipitation; IB, immunoblot. (F) SUMO-1 knockdown antagonized BetA-induced inhibition of Sp1 expression. SUMO-1 siRNA was transfected into CAL-27 cells for $24 \mathrm{~h}$ and then treated with BetA (10 $\mu \mathrm{M})$ for another $24 \mathrm{~h}$. Protein expression was detected by western blot analysis. (G) SUMO-1 was knocked down in CAL-27 cells, and then the cells was treated with radiation, BetA or a combination of radiation and BetA, and the cleaved caspase- 3 was detected using western blot analysis.

radiation-induced cell proliferation inhibition and apoptosis. Furthermore, we detected the level of cleaved caspase- 3 and found that treatment with radiation or BetA alone resulted in only a slight increase of cleaved caspase-3, while treatment with radiation after BetA treatment significantly increased cleaved caspase 3 (Fig. 2F and G).
Spl may be responsible for radioresistance of OSCC. Sp1 is reported to play a key role in radioresistance in several tumors. In order to reveal whether Sp1 is also involved in OSCC radioresistance, we detected the expression of Sp1 after radiation in CAL-27 cells and Tca-83 cells. As shown in Fig. 3A, Sp1 was dose-dependently upregulated by 
radiation in these cells, with a corresponding downregulation of its downstream target, PTEN. Furthermore, we knocked down Sp1 by transfecting Sp1 specific siRNA into these two cells (Fig. 3B), and we found that knockdown of Sp1 significantly antagonized radiation-induced PTEN downregulation (Fig. 3C), as well as facilitated radiationinduced inhibition of cell proliferation and apoptosis in CAL-27 and Tca-83 cells (Fig. 3D). The above suggested that Sp1 might be responsible for radioresistance of OSCC.

BetA antagonizes radiation-induced Spl upregulation and rescued PTEN expression. As Sp1 may be responsible for radioresistance of OSCC, we next detected whether BetA promoted radiosensitization through $\mathrm{Sp} 1$. We treated CAL-27 and Tca-83 cells with BetA, as shown in Fig. 4A, BetA downregulated $\mathrm{Sp} 1$ and upregulated PTEN in these cells. Moreover, we demonstrated that BetA antagonizes radiation-induced Sp1 overexpression, with a corresponding upregulation of PTEN (Fig. 4B).

BetA inhibits Spl expression through inducing Spl sumoylation. $\mathrm{Spl}$ protein stability may be regulated by several protein modifications. To explore the underlying mechanism of BetA regulating $\mathrm{Sp} 1$, we detected whether Sumoylation regulated Sp1 expression in CAL-27 cells. We overexpressed SUMO-1, an enzyme regulating sumoylation of several proteins. As shown in Fig. 5A, overexpression of SUMO-1 increased Sp1 sumoylation, as well as decreased Sp1 expression and increased PTEN expression (Fig. 5B). Whereas, knockdown of SUMO-1 using its specific siRNA downregulated Sp1 sumoylation (Fig. 5C) and correspondingly upregulated Sp1 and downregulated PTEN (Fig. 5D). We further investigated whether knockdown of SUMO-1 antagonized BetA-induced Sp1 sumoylation. The immunoprecipitation assay showed that BetA-induced Sp1 sumoylation, indicating that BetA promoted the interaction between SUMO-1 and Sp1, whereas knockdown of SUMO-1 could antagonize BetA-induced Sp1 sumoylation (Fig. 5E). Furthermore, western blot analysis showed that knockdown of SUMO-1 correspondingly antagonized BetA-induced Sp1 downregulation and the corresponding PTEN upregulation (Fig. 5F). Noteworthy, western blot analysis showed that BetA did not regulate the expression of SUMO-1 (Fig. 5F), thus, BetA only regulates the affinity of SUMO-1 to Sp1 but not the expression of SUMO-1. In addition, we knocked down SUMO-1 in CAL-27 cells, and detected whether knockdown of SUMO-1 impaired the BetA-induced radiosensitization. The results showed that BetA only slightly induced radiosensitization in the condition of SUMO-1 being knocked down, indicating that knockdown of SUMO-1 at least partially impaired the BetA-induced radiosensitization (Fig. 5G).

Sumoylation inhibits Spl binding to PTEN promoter. Since we demonstrated that BetA induced sumoylation of Sp1 and correspondingly induced Sp1 downregulation and PTEN overexpression, we investigated how sumoylation of Sp1 regulated PTEN. We transfected SUMO-1 into CAL-27 cells to induce Sp1 sumoylation and then performed ChIP assay. As shown in Fig. 6A, increase of Sp1 sumoylation inhibited Sp1 DNA-binding activity to PTEN promoter. Further, we also

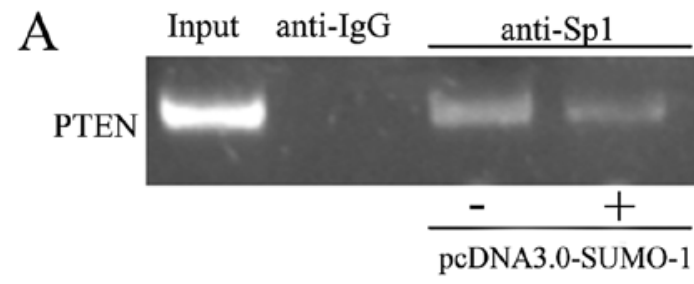

B

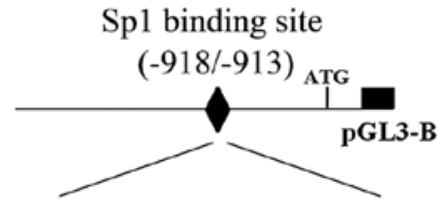

Wild-type CCGGCGGGCGGTGATG

Mutant type CCGGCGAACGGTGATG

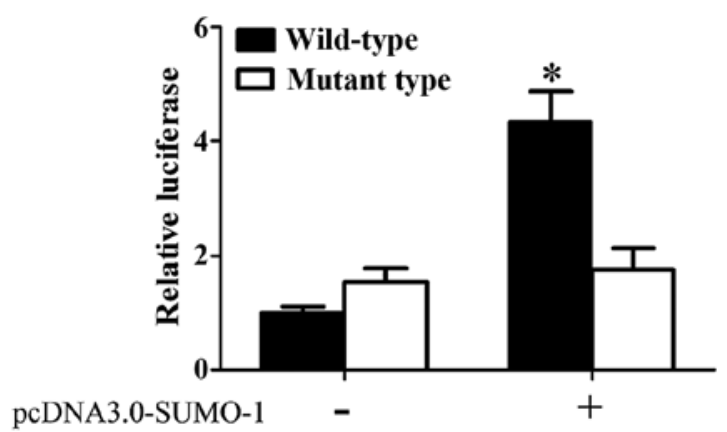

Figure 6. (A) Sumoylation inhibits Spl binding to PTEN promoter. ChIP assays were performed in CAL-27 cells with anti-Sp1 or anti-FLAG antibodies and with primers amplifying the $-1138 /-606$ region of the PTEN promoter (translation site ATG as +1 ) containing Sp1-binding site. (B) Sumoylation of Sp1 increased PTEN promoter activity. Upper panel shows the schematic map of PTEN promoter. The PTEN promoter is simulated by a straight line. The Sp1-binding site is represented by a filled diamond shape. The translational start site was defined as $(+1)$ because multiple transcriptional start sites between_958 and_821 before the initiation codon ATG were reported. The black box represents the luciferase reporter (pGL3-B, pGL3-Basic plasmid). Lower panel shows the data of luciferase assay. The reporter plasmid was transfected after pcDNA3.0-SUMO-1 being transfected for $24 \mathrm{~h}$. Luciferase activity was measured after CAL-27 cells were transfected with wild-type or mutated PTEN promoter-reporter constructs for $24 \mathrm{~h}$. ${ }^{*} \mathrm{P}<0.05$.

detected PTEN promoter activity. We found that overexpression of SUMO-1 decreased PTEN promoter activity, whereas, once we mutated Spl specific binding site in PTEN promoter, overexpression of SUMO-1 could not regulate the PTEN promoter (Fig. 6B), indicating that sumoylation regulated Sp1 DNA binding activity.

\section{Discussion}

In the present study, we proved for the first time that BetA induced radiosensitization in OSCC through inducing Sp1 sumoylation. First, BetA increased radiosensitization in OSCC. Second, Spl was upregulated after radiation and responsible for radioresistance, however, BetA could antagonize radiationinduced Sp1 overexpression. Third, BetA inhibited Sp1 expression through inducing Sp1 sumoylation, correspondingly inducing PTEN expression.

Overexpression of $\mathrm{Sp} 1$ mediates radioresistance in OSCC. Radioresistance is a huge impediment to radiotherapy (52). Cancer cells resistant to radiotherapy can result in therapy 
failure and the local recurrence $(53,54)$. Several pathways and genes have been reported to be involved in radioresistance including nuclear factor- $\kappa \mathrm{B}$ (NF- $\kappa \mathrm{B})$, signal transducer and activator of transcription 3 (STAT3), DNA-dependent protein kinase catalytic subunit (DNA-PKcs) and ataxia telangiectasia-mutated gene (ATM) (14-18). Our finding of Sp1 involved in regulating radiosensitization through regulating PTEN revealed a new mechanism of radioresistance in OSCC. PTEN inhibited AKT activation thus it is involved in three major radioresistance mechanisms: intrinsic radioresistance, tumor-cell proliferation, and hypoxia (55). Actually, Sp1 could interact with some other traditional molecules which could influence the outcome of radiotherapy, for example, $\mathrm{Sp} 1$ could transcriptionally regulate DNA-PKcs expression through binding to a GC-rich region in the promoter (56). The interactions of $\mathrm{Spl}$ with these molecules could be possible mechanisms for inducing radioresistance, however, our finding of Sp1 regulating PTEN at least partially involves in radioresistance. Our results suggested that $\mathrm{Sp1}$ could be a promising target for increasing radiosensitization in OSCC radiotherapy.

BetA enhanced radiosensitization at least partially by regulating Sp1/PTEN. We observed that BetA could significantly enhance radiosensitization of the two types of cancer cells. We also observed that BetA significantly inhibited Sp1 expression as well as induced PTEN expression. Thereby BetA could rescue radiation-induced Sp1 overexpression and PTEN inhibition. Considering that PTEN plays a crucial role in radiosensitization $(34,57,58)$, our results suggested that inhibition of Sp1 expression and induction of PTEN expression by BetA contributed to the radiosensitization of OSCC. Actually BetA and its derivatives have already been demonstrated to regulate radiosensitization $(50,51,59)$. Bache et al used Bet A at a concentration of $10 \mu \mathrm{M}$ and demonstrated that radiosensitization ability of BetA could be enhanced in hypoxic condition (51); our study agreed with theirs, while whether Sp1/PTEN pathway involves in hypoxic-induced radiosensitization ability enhancement of BetA is still unknown. Furthermore, there are also several mechanisms reveled underlying BetA-induced radiosensitization $(50,51,59)$. However, our funding could be another important mechanism underlying the BetA enhancement of radiosensitization besides the other previously reported mechanisms $(50,51,59)$, and further supported the assumption that BetA could be a clinically available and promising enhancer of tumor radiotherapy. According to our results, the enhancement of radiosensitization by BetA could be compromised in tumors with aberrant PTEN. Further studies are needed to evaluate how much the enhancement on radiosensitization by BetA would be compromised in PTEN-null cells. Nevertheless, our results have clinical relevance because the application of BetA as an enhancer for radiotherapy may be more suitable for tumors whose PTEN was not mutated or deleted.

BetA upregulated PTEN protein expression partially by inhibiting Sp1 expression, thereby activated PTEN transcription. Several transcription factor regulating PTEN have been identified, including NF- $\kappa$ B, Egr-1, p53 and Sp1 $(26,60,61)$. Spl downregulates PTEN expression through binding to a specific site (26). Our study showed that BetA inhibited Sp1 sumoylation and correspondingly upregulated PTEN expression through inhibiting Sp1 expression and DNA-binding activity. Considering that we have previously demonstrated that only acetylated Sp1 could regulated PTEN expression (26), so whether the two post-translational modifications, sumoylation and acetylation, could be inter-regulated by each other or have competition needs further study. There are already several reports on interaction of sumoylation and acetylation, as Kim et al reported that acetylation of FXR at site K217 inhibited the sumoylation of FXR at K227 (62). Therefore, it is possible that there is a mutual regulation of Sp1 sumoylation and acetylation. Furthermore, BetA was reported to affect $\mathrm{Sp} 1$ sumoylation through sentrin-specific protease 1. Our study demonstrated BetA regulating Sp1 sumoylation while revealing a new mechanism. Although, which mechanism is more important remains unknown, these two studies do suggest BetA could be a promising anticancer drug. Sumoylation of $\mathrm{Sp} 1$ has already been reported to be affected by BetA (63), our study agreed their conclusion and further illustrated that BetA regulated PTEN through Sp1 sumoylation and correspondingly induced radiosensitization. In addition, $\mathrm{Sp} 1$ was reported to have two sumoylation sites, K16 and K683 (28), but in treatment with BetA, which one was sumoylated remains unknown, so further study to confirm which one was involved in BetA-induced Sp1 sumoylation is required.

There are several limitation as some questions are still unclear: 1) sumoylation regulates Sp1 binding to PTEN promoter, but the underlying mechanism needs to be revealed; 2) radiosensitization ability of BetA could be enhanced in hypoxic condition (51); however, whether Sp1/PTEN pathway is involved in this process is unknown. These are very interesting questions, and we will continue their clarification. Further animal study and preclinical studies are needed to support our conclusion.

In conclusion, we showed that BetA blocked radiationinduced Sp1 overexpression and PTEN downregulation through inducing Spl sumoylation and thereby contributed to radiosensitization of OSCC.

\section{Acknowledgements}

This work was supported by the National Natural Science Foundation of China (grant no. 81272958 and 81472530).

\section{References}

1. Torre LA, Bray F, Siegel RL, Ferlay J, Lortet-Tieulent J and Jemal A: Global cancer statistics, 2012. CA Cancer J Clin 65: 87-108, 2015.

2. Leemans CR, Braakhuis BJ and Brakenhoff RH: The molecular biology of head and neck cancer. Nat Rev Cancer 11: 9-22, 2011.

3. Siegel RL, Miller KD and Jemal A: Cancer statistics, 2016. CA Cancer J Clin 66: 7-30, 2016.

4. Chen W, Zheng R, Baade PD, Zhang S, Zeng H, Bray F, Jemal A, Yu XQ and He J: Cancer statistics in China, 2015. CA Cancer J Clin 66: 115-132, 2016.

5. Mao L: Oral squamous cell carcinoma - progresses from risk assessment to treatment. Chin J Dent Res 15: 83-88, 2012.

6. Wolchok JD and Chan TA: Cancer: Antitumour immunity gets a boost. Nature 515: 496-498, 2014.

7. Mahoney KM, Rennert PD and Freeman GJ: Combination cancer immunotherapy and new immunomodulatory targets. Nat Rev Drug Discov 14: 561-584, 2015.

8. Baumann M, Krause M, Overgaard J, Debus J, Bentzen SM, Daartz J, Richter C, Zips D and Bortfeld T: Radiation oncology in the era of precision medicine. Nat Rev Cancer 16: 234-249, 2016. 
9. Martin NE and D'Amico AV: Progress and controversies: Radiation therapy for prostate cancer. CA Cancer J Clin 64: 389-407, 2014.

10. Jones RM, Sloane VM, Wu H, Luo L, Kumar A, Kumar MV, Gewirtz AT and Neish AS: Flagellin administration protects gut mucosal tissue from irradiation-induced apoptosis via MKP-7 activity. Gut 60: 648-657, 2011.

11. Jun S, Jung YS, Suh HN, Wang W, Kim MJ, Oh YS, Lien EM, Shen X, Matsumoto Y, McCrea PD, et al: LIG4 mediates Wnt signalling-induced radioresistance. Nat Commun 7: 10994, 2016.

12. Advani SJ, Camargo MF, Seguin L, Mielgo A, Anand S, Hicks AM, Aguilera J, Franovic A, Weis SM and Cheresh DA: Kinase-independent role for CRAF-driving tumour radioresistance via CHK2. Nat Commun 6: 8154, 2015.

13. Fu HC, Yang YC, Chen YJ, Lin H, Ou YC, Chien CC, Huang EY, Huang HY, Lan J, Chi HP, et al: Increased expression of SKP2 is an independent predictor of locoregional recurrence in cervical cancer via promoting DNA-damage response after irradiation. Oncotarget 7: 44047-44061, 2016

14. Bai M, Ma X, Li X, Wang X, Mei Q, Li X, Wu Z and Han W: The accomplices of NF- $\kappa$ B lead to radioresistance. Curr Protein Pept Sci 16: 279-294, 2015.

15. Ahmed KM, Zhang $\mathrm{H}$ and Park $\mathrm{CC}$ : $\mathrm{NF}-\kappa \mathrm{B}$ regulates radioresistance mediated by $\beta 1$-integrin in three-dimensional culture of breast cancer cells. Cancer Res 73: 3737-3748, 2013.

16. Aggarwal BB and Sung B: NF- $\kappa$ B in cancer: A matter of life and death. Cancer Discov 1: 469-471, 2011.

17. Zhang P, Wei Y, Wang L, Debeb BG, Yuan Y, Zhang J, Yuan J, Wang M, Chen D, Sun Y, et al: ATM-mediated stabilization of ZEB1 promotes DNA damage response and radioresistance through CHK1. Nat Cell Biol 16: 864-875, 2014.

18. Wang L, Yang H, Palmbos PL, Ney G, Detzler TA, Coleman D, Leflein J, Davis M, Zhang M, Tang W, et al: ATDC/TRIM29 phosphorylation by ATM/MAPKAP kinase 2 mediates radioresistance in pancreatic cancer cells. Cancer Res 74: 1778-1788, 2014.

19. Wang J, Kang M, Qin YT, Wei ZX, Xiao JJ and Wang RS: Sp1 is over-expressed in nasopharyngeal cancer and is a poor prognostic indicator for patients receiving radiotherapy. Int J Clin Exp Pathol 8: 6936-6943, 2015.

20. Kang M, Xiao J, Wang J, Zhou P, Wei T, Zhao T and Wang R: MiR-24 enhances radiosensitivity in nasopharyngeal carcinoma by targeting SP1. Cancer Med 5: 1163-1173, 2016.

21. Enomoto A, Fukasawa T, Takamatsu N, Ito M, Morita A Hosoi Y and Miyagawa K: The HSP90 inhibitor 17-allylamino17-demethoxygeldanamycin modulates radiosensitivity by downregulating serine/threonine kinase 38 via Sp1 inhibition. Eur J Cancer 49: 3547-3558, 2013

22. Cawley S, Bekiranov S, Ng HH, Kapranov P, Sekinger EA, Kampa D, Piccolboni A, Sementchenko V, Cheng J, Williams AJ, et al: Unbiased mapping of transcription factor binding sites along human chromosomes 21 and 22 points to widespread regulation of noncoding RNAs. Cell 116: 499-509, 2004

23. Zannetti A, Del Vecchio S, Carriero MV, Fonti R, Franco P, Botti G, D'Aiuto G, Stoppelli MP and Salvatore M: Coordinate up-regulation of Sp1 DNA-binding activity and urokinase receptor expression in breast carcinoma. Cancer Res 60 1546-1551, 2000.

24. Vizcaíno C, Mansilla S and Portugal J: Sp1 transcription factor: A long-standing target in cancer chemotherapy. Pharmacol Ther 152: 111-124, 2015.

25. Wang L, Wei D, Huang S, Peng Z, Le X, Wu TT, Yao J, Ajani J and Xie K: Transcription factor $\mathrm{Spl}$ expression is a significant predictor of survival in human gastric cancer. Clin Cancer Res 9: 6371-6380, 2003.

26. Kou XX, Hao T, Meng Z, Zhou YH and Gan YH: Acetylated Spl inhibits PTEN expression through binding to PTEN core promoter and recruitment of HDAC1 and promotes cancer cell migration and invasion. Carcinogenesis 34: 58-67, 2013.

27. Yang HC, Chuang JY, Jeng WY, Liu CI, Wang AH, Lu PJ, Chang WC and Hung JJ: Pin1-mediated Spl phosphorylation by CDK1 increases Sp1 stability and decreases its DNA-binding activity during mitosis. Nucleic Acids Res 42: 13573-13587, 2014.

28. Gong L, Ji WK, Hu XH, Hu WF, Tang XC, Huang ZX, Li L, Liu M, Xiang SH, Wu E, et al: Sumoylation differentially regulates Sp1 to control cell differentiation. Proc Natl Acad Sci USA 111: 5574-5579, 2014
29. Lee HJ, Ryu JM, Jung YH,Lee KH, Kim DI and Han HJ: Glycerol3-phosphate acyltransferase-1 upregulation by O-GlcNAcylation of Spl protects against hypoxia-induced mouse embryonic stem cell apoptosis via mTOR activation. Cell Death Dis 7: e2158, 2016.

30. Chang WC and Hung JJ: Functional role of post-translational modifications of Sp1 in tumorigenesis. J Biomed Sci 19: 94, 2012.

31. Steck PA, Pershouse MA, Jasser SA, Yung WK, Lin H, Ligon AH, Langford LA, Baumgard ML, Hattier T, Davis T, et al: Identification of a candidate tumour suppressor gene, MMAC1, at chromosome $10 \mathrm{q} 23.3$ that is mutated in multiple advanced cancers. Nat Genet 15: 356-362, 1997.

32. Maehama T and Dixon JE: The tumor suppressor, PTEN/ MMAC1, dephosphorylates the lipid second messenger, phosphatidylinositol 3,4,5-trisphosphate. J Biol Chem 273: 13375-13378, 1998.

33. Li J, Yen C, Liaw D, Podsypanina K, Bose S, Wang SI, Puc J, Miliaresis C, Rodgers L, McCombie R, et al: PTEN, a putative protein tyrosine phosphatase gene mutated in human brain, breast, and prostate cancer. Science 275: 1943-1947, 1997.

34. Meng Z and Gan YH: Activating PTEN by COX-2 inhibitors antagonizes radiation-induced $\mathrm{AKT}$ activation contributing to radiosensitization. Biochem Biophys Res Commun 460: 198-204, 2015.

35. Zheng L, Zhang Y, Liu Y, Zhou M, Lu Y, Yuan L, Zhang C, Hong M, Wang S and Li X: MiR-106b induces cell radioresistance via the PTEN/PI3K/AKT pathways and p21 in colorectal cancer. J Transl Med 13: 252, 2015.

36. Bardia A, Keenan TE, Ebbert JO, Lazovich D, Wang AH, Vierkant RA, Olson JE, Vachon CM, Limburg PJ, Anderson KE, et al: Personalizing aspirin use for targeted breast cancer chemoprevention in postmenopausal women. Mayo Clin Proc 91: 71-80, 2016.

37. Nimptsch K, Zhang X, Cassidy A, Song M, O'Reilly ÉJ, Lin JH, Pischon T, Rimm EB, Willett WC, Fuchs CS, et al: Habitual intake of flavonoid subclasses and risk of colorectal cancer in 2 large prospective cohorts. Am J Clin Nutr 103: 184-191, 2016.

38. He E, Pan F, Li G and Li J: Fractionated ionizing radiation promotes epithelial-mesenchymal transition in human esophageal cancer cells through PTEN deficiency-mediated Akt activation. PLoS One 10: e0126149, 2015.

39. Lo YC, Chang YH, Wei BL, Huang YL and Chiou WF: Betulinic acid stimulates the differentiation and mineralization of osteoblastic MC3T3-E1 cells: Involvement of BMP/Runx2 and beta-catenin signals. J Agric Food Chem 58: 6643-6649, 2010.

40. Lingaraju MC, Pathak NN, Begum J, Balaganur V, Bhat RA, Ram M, Kumar D, Kumar D and Tandan SK: Betulinic acid negates oxidative lung injury in surgical sepsis model. J Surg Res 193: 856-867, 2015.

41. Alakurtti S, Mäkelä T, Koskimies S and Yli-Kauhaluoma J: Pharmacological properties of the ubiquitous natural product betulin. Eur J Pharm Sci 29: 1-13, 2006.

42. Ehrhardt H, Fulda S, Führer M, Debatin KM and Jeremias I: Betulinic acid-induced apoptosis in leukemia cells. Leukemia 18: 1406-1412, 2004

43. Tan Y, Yu R and Pezzuto JM: Betulinic acid-induced programmed cell death in human melanoma cells involves mitogen-activated protein kinase activation. Clin Cancer Res 9: 2866-2875, 2003.

44. Pisha E, Chai H, Lee IS, Chagwedera TE, Farnsworth NR, Cordell GA, Beecher CW, Fong HH, Kinghorn AD, Brown DM, et al: Discovery of betulinic acid as a selective inhibitor of human melanoma that functions by induction of apoptosis. Nat Med 1: 1046-1051, 1995.

45. Zhang DM, Xu HG, Wang L, Li YJ, Sun PH, Wu XM, Wang GJ, Chen WM and Ye WC: Betulinic acid and its derivatives as potential antitumor agents. Med Res Rev 35: 1127-1155, 2015

46. Li L, Du Y, Kong X, Li Z, Jia Z, Cui J, Gao J, Wang G and Xie K: Lamin B1 is a novel therapeutic target of betulinic acid in pancreatic cancer. Clin Cancer Res 19: 4651-4661, 2013

47. Gao Y, Jia Z, Kong X, Li Q, Chang DZ, Wei D, Le X, Suyun H, Huang S, Wang L, et al: Combining betulinic acid and mithramycin a effectively suppresses pancreatic cancer by inhibiting proliferation, invasion, and angiogenesis. Cancer Res 71: 51825193, 2011.

48. Kasperczyk H, La Ferla-Brühl K, Westhoff MA, Behrend L, Zwacka RM, Debatin KM and Fulda S: Betulinic acid as new activator of NF-kappaB: Molecular mechanisms and implications for cancer therapy. Oncogene 24: 6945-6956, 2005. 
49. Zuco V, Supino R, Righetti SC, Cleris L, Marchesi E, Gambacorti-Passerini C and Formelli F: Selective cytotoxicity of betulinic acid on tumor cell lines, but not on normal cells. Cancer Lett 175: 17-25, 2002.

50. Bache M, Bernhardt S, Passin S, Wichmann H, Hein A, Zschornak M, Kappler M, Taubert H, Paschke R and Vordermark D: Betulinic acid derivatives NVX-207 and B10 for treatment of glioblastoma - an in vitro study of cytotoxicity and radiosensitization. Int J Mol Sci 15: 19777-19790, 2014.

51. Bache M, Zschornak MP, Passin S, Kessler J, Wichmann H, Kappler M, Paschke R, Kaluđerović GN, Kommera H, Taubert H, et al: Increased betulinic acid induced cytotoxicity and radiosensitivity in glioma cells under hypoxic conditions. Radiat Oncol 6 : $111,2011$.

52. Bernier J: Current state-of-the-art for concurrent chemoradiation. Semin Radiat Oncol 19: 3-10, 2009.

53. Lee S, Lim MJ, Kim MH, Yu CH, Yun YS, Ahn J and Song JY: An effective strategy for increasing the radiosensitivity of Human lung Cancer cells by blocking Nrf2-dependent antioxidant responses. Free Radic Biol Med 53: 807-816, 2012.

54. Provencio M, Sánchez A, Garrido P and Valcárcel F: New molecular targeted therapies integrated with radiation therapy in lung cancer. Clin Lung Cancer 11: 91-97, 2010.

55. Bussink J, van der Kogel AJ and Kaanders JH: Activation of the PI3-K/AKT pathway and implications for radioresistance mechanisms in head and neck cancer. Lancet Oncol 9: 288-296, 2008.

56. Nishida Y, Mizutani N, Inoue M, Omori Y, Tamiya-Koizumi K, Takagi A, Kojima T, Suzuki M, Nozawa Y, Minami Y, et al: Phosphorylated $\mathrm{Sp} 1$ is the regulator of DNA-PKcs and DNA ligase IV transcription of daunorubicin-resistant leukemia cell lines. Biochim Biophys Acta 1839: 265-274, 2014.
57. Zhang Y, Zheng L, Ding Y, Li Q, Wang R, Liu T, Sun Q, Yang H, Peng S, Wang W, et al: MiR-20a induces cell radioresistance by activating the PTEN/PI3K/Akt signaling pathway in hepatocellular carcinoma. Int J Radiat Oncol Biol Phys 92: 1132-1140, 2015.

58. Liu ZL, Wang H, Liu J and Wang ZX: MicroRNA-21 (miR-21) expression promotes growth, metastasis, and chemo- or radioresistance in non-small cell lung cancer cells by targeting PTEN. Mol Cell Biochem 372: 35-45, 2013.

59. Eder-Czembirek C, Erovic BM, Czembirek C, Brunner M, Selzer E, Pötter R and Thurnher D: Betulinic acid a radiosensitizer in head and neck squamous cell carcinoma cell lines. Strahlenther Onkol 186: 143-148, 2010.

60. Stambolic V, MacPherson D, Sas D, Lin Y, Snow B, Jang Y, Benchimol S and Mak TW: Regulation of PTEN transcription by p53. Mol Cell 8: 317-325, 2001.

61. Virolle T, Adamson ED, Baron V, Birle D, Mercola D, Mustelin T and de Belle I: The Egr-1 transcription factor directly activates PTEN during irradiation-induced signalling. Nat Cell Biol 3: 1124-1128, 2001.

62. Kim DH, Xiao Z, Kwon S, Sun X, Ryerson D, Tkac D, Ma P, Wu SY, Chiang CM, Zhou E, et al: A dysregulated acetyl/SUMO switch of FXR promotes hepatic inflammation in obesity. EMBO J 34: 184-199, 2015.

63. Hsu TI, Wang MC, Chen SY, Huang ST, Yeh YM, Su WC, Chang WC and Hung JJ: Betulinic acid decreases specificity protein $1(\mathrm{Sp} 1)$ level via increasing the sumoylation of $\mathrm{sp} 1$ to inhibit lung cancer growth. Mol Pharmacol 82: 1115-1128, 2012. 